\title{
Associations between Physical Activity Motives and Trends in Moderate-to-Vigorous Physical Activity Among Adolescents Over Five Years.
}

\section{Patrick Abi Nader}

Centre de formation médicale du Nouveau-Brunswick https://orcid.org/0000-0003-2308-6372

\section{Jeffrey Gaudet}

Centre de formation médicale du Nouveau-Brunswick

Jennifer Brunet

University of Ottawa

Katie E. Gunnell

Carleton University

Isabelle Doré

Universite de Montreal

Catherine M. Sabiston

University of Toronto

Jonathan Boudreau

University of New Brunswick

Mathieu Bélanger ( $\square$ mathieu.f.belanger@usherbrooke.ca )

Centre de formation médicale du Nouveau-Brunswick https://orcid.org/0000-0001-9446-6538

\section{Research}

Keywords: Longitudinal, Measurement invariance, Moderate-to-vigorous, Reasons

Posted Date: December 12th, 2019

DOI: https://doi.org/10.21203/rs.2.18642/v1

License: (c) (1) This work is licensed under a Creative Commons Attribution 4.0 International License. Read Full License 


\section{Abstract}

\section{BACKGROUND}

Motives for participation in physical activity (PA) have been associated with moderate-to-vigorous intensity PA (MVPA); however, these associations have yet to be established during the transition from childhood to adolescence. This study examined the longitudinal associations between five PA motives (i.e., enjoyment, competence, fitness, social, and appearance) and MVPA over a 5-year period spanning late childhood (9-11 years), early adolescence (12-14 years), and middle adolescence (15-16 years).

\section{METHODS}

Self-report data $(n=937)$ were drawn from the ongoing Monitoring Activities for Teenagers to Comprehend their Habits study. The Motives for Physical Activity Measure-Revised (MPAM-R) questionnaire was used to measure PA motives. MVPA was assessed with an empirically supported 2item measure developed specifically for adolescents. Data from three times points per school year over 5 years (resulting in 15 survey cycles) were analyzed using sex-stratified mixed effects regression models after establishing longitudinal measurement invariance for the MPAM-R.

\section{RESULTS}

MVPA increased from cycle 1 (9-11 years) to 7 (11-13 years) for girls and from cycle 1 (9-11 years) to 9 (12-14 years) for boys before decreasing for both sexes through to cycle 15 (14-16 years). Longitudinal measurement invariance was established for a 22-item version of the MPAM-R. For boys, enjoyment and competence motives were positively associated with MVPA from late childhood to middle adolescence and fitness motives were negatively associated with MVPA from late childhood to middle adolescence. For girls, enjoyment motives were positively associated with MVPA during late childhood and competence motives were positively associated with MVPA from late childhood to middle adolescence.

\section{CONCLUSIONS}

PA interventions should aim to increase enjoyment during PA and provide opportunities for children and adolescents to develop a desire to build competence during PA. PA interventions targeted to youth should avoid promoting the desire to be active to improve fitness, particularly among boys.

\section{Background}

Despite being associated with a plethora of health benefits, [1-5], including preventing early onset of chronic diseases and increasing life expectancy [5-9], low levels of physical activity (PA) prevail among youth. [10] Whilst interventions aimed at increasing PA during adolescence have had limited short-term effects, [11-14] there is a need to understand factors that explain long-term moderate-to-vigorous intensity PA (MVPA) trends in youth to inform behavior change strategies. The focus on youth is 
especially important given that the transition from late childhood to adolescence is a development time period with well-documented declines in PA. $[15,16]$

PA motives, commonly referred to as the reasons for engaging in PA, have been well-studied predictors of participation across the lifespan. Based on a number of behavioral theories (e.g., theory of planned behaviour, [17] social ecological model, [18] self-efficacy theory, [19] basic psychological needs theory, [20] expectancy-value model, [21]), competence, enjoyment, and social motives are associated with PA participation. Further to these prominent motives, it has also been suggested that individuals engage in PA for appearance and health/fitness reasons. [22, 23] Overall, PA motives associated with enjoyment and competence may be more strongly associated with maintenance of PA because of their more intrinsic nature, [23-28], compared to fitness, appearance, and social motives which are more extrinsic motives. $[23,26,27]$.

However, empirical support for these proposed associations among youth has been mixed. In a British sample of middle adolescents (ages 13-15 years; $n=580$ ), a cross-sectional study showed that fitness and enjoyment motives were associated with greater leisure-time PA, whereas appearance motives were associated with lower self-reported PA. [29] In another cross-sectional study with a Spanish sample of adolescents (mean age of 15 years; $n=1098$ ), Chicote-López et al. [30] reported that enjoyment, competence, fitness, and social motives were all associated with greater self-reported PA; appearance motives were not assessed. Similarly, among children ages $10-12$ years $(n=1272)$ from Belgium, a composite score representing fitness, competence, and social (affiliation) motives was indirectly positively associated with daily step counts measured with a pedometer, whereas a composite score of appearance and social (recognition) motives was indirectly negatively associated with daily step counts. [31] Enjoyment during PA was measured in the study but was not included as a motive for PA in associative models. [31] In a similar age group, a longitudinal study with two measurement points with children ages $10-12$ years ( $n=1081$ in fifth grade, and $n=1011$ in sixth grade) from the United States of America (USA) found that enjoyment and competence motives were positively associated with accelerometer-measured MVPA. [32] Moreover, appearance, social, and fitness motives were not related to PA. [32] In a cross-sectional analysis with 802 Canadian children (mean age 10.7) members of our research group found that competence and enjoyment motives were significantly associated with PA but neither social, appearance, and fitness motives were associated with PA. [33] Specifically, Goguen et al. [33] found that enjoyment motives were associated with greater likelihood of participating in organized PA, and that competence motives were associated with greater likelihood of meeting PA guidelines and of participating in group-based PA. Additionally, in a small cross-sectional study of children (ages 7-12 years; $n=49$ ) from the USA, enjoyment motives were ranked as a primary reason for being active, followed by competence, fitness, and social motives. [34] Given that PA motives are inconsistently related to youth PA across studies and that studies sample youth of varying ages, it is challenging to identify which motives should be fostered to facilitate PA behaviour change and maintenance.

Previous studies have also found differences in the associations between PA motives and PA between boys and girls. For example, in one cross-sectional study, [35] social motives were positively related with 
PA for girls only, fitness motives were positively associated with PA for boys only, and competence motives were positively associated with PA for both sexes. In contrast, another cross-sectional study [36] found that boys participated in sports for social motives, whereas girls participated in sports for fitness motives. Additionally, other cross-sectional studies have found that social and appearance motives were positively associated with PA for girls only. $[37,38]$ The conflicting findings regarding which motive(s) was/were more important for PA prediction among boys and girls, and the lack of longitudinal studies examining how these relationships change during the transition from late childhood to adolescence underscore the need to address these gaps in the literature with a longitudinal study with frequent measurements.

As a first step, however, it is necessary to ensure the measure used to assess PA motives can be used during late childhood and adolescence. In previous studies, PA motives have been measured using different questionnaires including the Motives for Physical Activity Measure-Revised (MPAM-R) questionnaire, $[23,27]$ which received little attention despite measuring five motives with 30 items, [23, 27, 28] rather than 18 or less as is done with other measures. [39-41] To date, longitudinal invariance of the MPAM-R was tested with two measurement cycles with children (boys and girls) transitioning from grade 5 to grade 6. [32] Therefore, its longitudinal invariance has not been examined from late childhood to adolescence (i.e. transition through two distinctive life periods) among boys and girls, and is warranted in this study.

Given all aforementioned gaps and inconsistent findings, the primary aim of this investigation was to examine the longitudinal associations between PA motives and MVPA over a 5-year period spanning the transition from late childhood (9-11 years), early adolescence (12-14 years), to middle adolescence (15-16 years) for boys and girls. Using a longitudinal design to examine these associations permitted the assessment of if and how these relationships changed over time. A secondary aim was to establish longitudinal measurement invariance for the MPAM-R.

\section{Methods}

Data were derived from the longitudinal "Monitoring Activities of Teenagers to Comprehend their Habits" (MATCH) study. [42] At study inception, English- and French-speaking grade 5 and grade 6 students (9-12 years) were recruited from 17 schools in New Brunswick, Canada. Schools were situated in a mix of rural and urban areas representing diverse socio-economic backgrounds. Participants completed self-report questionnaires three times per year (i.e., fall, winter, and spring) at four month intervals every year until graduating from Grade 12 (16-18 years). These time points were specifically chosen to control for the potential confounding effects of seasonality (i.e., fall, winter, spring). [43]

In the first year of data collection, there were 806 participants (55\% girls; $65 \%$ French-speaking) who were, on average, $10.3 \pm 0.6$ years of age. In addition, 131 students from participating schools joined after the first year of the MATCH study, such that a total of 937 participants ( $56 \%$ girls; $66 \%$ French-speaking) took 
part in at least one survey cycle. Data on PA motives and PA were collected during cycles 1 to 15 which spanned the first 5 years of the MATCH study; analyses were restricted to data from those 15 cycles.

\section{PA Motives}

Participants' PA motives were assessed using the MPAM-R scale. $[23,27]$ Participants were asked to report the extent to which 30 items representing PA motives were true for them using a 7-point Likert-type scale, with 1 representing "not at all true for me" and 7 representing "very true for me." In theory, the MPAM-R includes 7 items assessing enjoyment motives (e.g. "because it makes me happy"), 7 items assessing competence motives (e.g. "because I want to improve existing skills"), 5 items assessing social motives (e.g. "because I want to be with friends"), 5 items assessing fitness motives (e.g. "because / want be physically fit"), and 6 items assessing appearance motives (e.g. "because I want to lose or maintain weight to look better'). The MPAM-R has demonstrated score reliability and validity in other studies. [32, 34]

\section{MVPA}

MVPA was assessed with a 2-item measure developed specifically for adolescents. [44] Participants were asked to read the following statement: "Physical activity is an activity that increases your heart rate and makes you get out of breath some of the time. Physical activity can be done in sports, playing with friends, or walking to school. Some examples of physical activity are running, brisk walking, rollerblading, biking, dancing, skateboarding, swimming, soccer, basketball, football, and surfing" and then asked: "Over the course of the week (past 7 days), how many days were you physically active for a total of at least 60 minutes per day?" and "Over the course of a typical or usual week, how many days are you physically active for a total of at least 60 minutes per day?" Response options ranged from 0 to 7 days. The two items were averaged to estimate a weekly average MVPA score. In previous work, this method to create MVPA scores was significantly correlated $(r=0.40, p<0.001)$ with accelerometer data, had an interclass correlation of 0.77 [44] and was supported for use among children and adolescents. [45-47]

\section{Data Analyses}

Descriptive statistics. Data were summarized to describe the participants at study inception and their evolution at every cycle. Means and ranges were computed to describe the age of participants. The median was calculated to describe the typical number of days reported for participating in at least 60 minutes of MVPA. Average scores and standard deviations for each PA motive were also calculated using the average of all subscale items.

Factor structure of the MPAM-R. The hypothesized correlated five-factor structure of the MPAM-R was verified with confirmatory factor analysis (CFA) using the 30 items at every survey cycle (i.e. 15 independent CFAs were computed). The computation was repeated twice; once with full information maximum likelihood (FIML) estimation to adjust the likelihood function so that each case contributes information on the variables that are observed, and a second time by using a robust estimation of the 
errors in addition to FIML (i.e. the Huber/White/Sandwich estimator). The items were set to load onto their corresponding factor as identified by Ryan et al. [23]. As the chi-square statistic has a tendency to reject the null hypothesis when the sample is large, model fit was evaluated with approximate methods. Specifically, model fit was considered acceptable when a Root Mean Square Error of Approximation (RMSEA) $<0.08$, a Comparative Fit Index $(\mathrm{CFI}) \geq 0.90$, and a Tucker-Lewis Index $(\mathrm{TLI}) \geq 0.90$ were obtained at every cycle. [48] If acceptable model fit was not achieved at least at one cycle, standardized loadings, modification indices, standardized residuals, squared multiple correlations, and covariances between items were scrutinized to determine if lack of acceptable model fit was due to a problematic item or a function of the hypothesized factor structure. Items that cross-loaded on factors not identified by the original authors of the MPAM-R, [23] were dropped from subsequent re-specified models until acceptable model fit was achieved at all cycles. In every re-specified model only one item at a time was dropped in the computation of the 15 CFAs corresponding to each cycle (e.g. after the first estimation of CFAs item 8 was identified as cross-loading, then item 8 was dropped from the next iteration of CFAs). Once an item was dropped it was not brought back to subsequent computations of CFAs.

Score reliability. To create the variables used for the main analysis in this study, five PA motives scores were calculated at each survey cycle using the mean of items retained for each of the motives (i.e., enjoyment, competence, social, fitness and appearance) in the final and reduced CFA model. Cronbach's alpha was calculated once using all items (i.e. all original items from the MPAM-R) and a second time with the retained items from the reduced CFA. This was completed to assess score reliability on the originally hypothesized variables as well as the variables informed from the reduced CFAs, respectively. Additionally, composite reliability was calculated using the "relicoef" command in Stata [49] that is based on Raykov's computation of reliability coefficients. [50]

Longitudinal Invariance. To ensure that the results reflected true change over time and not change in the psychometric structure of MPAM-R scores, the factor structure and measurement invariance over time was examined. [51] Four levels of invariance were estimated with the truncated MPAM-R using maximum likelihood parameter estimates with standard errors and a chi-square test statistic that are robust to nonnormality in Mplus: [52] (1) configural invariance (i.e., no equality constraints), (2) weak invariance (i.e., factor loadings constrained to be equal), (3) strong invariance (i.e., factor loadings and intercepts constrained to be equal), and (4) strict invariance (i.e., factor loadings, intercepts, and errors constrained to be equal). [51] Following Chen's [53] and Cheung's [54] recommendations, a change of $\leq 0.010$ in CFI supplemented by a change of $\leq 0.015$ in RMSEA was used as an indicator of invariance at each level.

Trajectories of PA Motives. The slopes and intercepts for each PA motive were estimated with mixed effects regression models using data from all 15 cycles. Both linear and quadratic fixed and random effects for cycle were tested in order to establish the functional form of change in PA motives. The quadratic term was retained for further analyses when it was statistically significant at $p<0.05$. Interactions for sex with time for each PA motive were tested and if they were significant for at least one of the motives, further analyses involving PA motives trajectories were stratified by sex. After, deciding on 
final models, variables representing the slopes and intercepts for each PA motive were generated so that they could be used in the primary analysis.

Trajectories of MVPA. Similar to PA motives, trajectories for MVPA were estimated with mixed effects regression models using all 15 cycles. Linear and quadratic fixed and random effects for cycle were also examined, and quadratic terms were retained if they were statistically significant at $p<0.05$. Sex interactions with time were tested and if significant, further analyses involving MVPA trajectories were stratified by sex.

Trajectories of PA motives and MVPA. To address the main objective of this study, mixed effects regression models were used to examine longitudinal associations between slopes and intercepts of each PA motive and MVPA using all 15 cycles. Variables representing the slopes and intercepts for each PA motive were used as independent variables in the same model (i.e. the effect of one PA motive was adjusted for all other PA motives). Sex interactions were tested and if statistically significant, further analyses of the relationship between PA motives and MVPA were stratified by sex.

Longitudinal invariance testing was conducted using Mplus 7.4, [52] whereas all other analysis (i.e., CFAs, mixed effects regression models) were estimated using Stata MP 15.1. [49] In all mixed effects regression models, the unstructured matrix was used to estimate the covariance matrix.

\section{Results}

Descriptive Statistics. These analyses were formed with 418 boys and 517 girls (Table 1). These participants were included in the final analysis since they participated in at least one measurement of self-reported MVPA and MPAM-R items that were used to compute each of the 5 motives. At cycle 1 , participants $(n=608)$ were on average 10.3 years old (range 9-12) and they reported participating in at least 60 minutes of MVPA on a median of 4.5 days per week. Moreover, boys reported a median of 5 days per week of achieving at least 60 minutes of MVPA, whereas girls reported a median of 4 days per week of achieving at least 60 minutes of MVPA. At every cycle, enjoyment motives received the highest mean score, followed by fitness, competence, social, and appearance motives respectively (Figure 1). Missing data for the outcome (i.e., MVPA) were very low, with a 98.1\% (range 93.2-99.8) average completion rate. Missing data for PA motives was larger and more variable by cycle, the average rate of complete data for the MPAM-R (i.e., responses available for the 30 items) was $84.1 \%$ (range 62.9-91.0). However, the average rate of having missing data on all 30 items from the MPAM-R was $1.02 \%$ (range $0-4.8$ ).

Factor structure of MPAM-R scores. The individual CFAs using the original 30 items as recommended by the authors of MPAM-R were unacceptable at each cycle. The average RMSEA was 0.08 (range 0.060.10), the average CFI was 0.87 (range $0.84-0.90$ ), and the average TLI was 0.86 (range 0.83-0.88). After multiple iterations in which one item at a time was dropped from all cycles due to cross-loadings on other factors, the final truncated MPAM-R included 22 items (i.e., the same 8 items were dropped from all cycles; see supplementary Table 1 for more details). The truncated MPAM-R yielded acceptable model fit in individual CFAs across all 15 cycles. The average RMSEA was 0.07 (range 0.05-0.08), the average CFI 
was 0.93 (range 0.91-0.95), and the average TLI was 0.92 (range 0.90-0.95). The final model resulted in the retention of five items representing fitness, four items for enjoyment, three items for competence, six items for appearance, and four items for social motives (see supplemental Table 1 for final retained items per subscale).

Score Reliability. Cronbach's alphas across all cycles for the original MPAM-R scale with all 30 items ranged between 0.76 and 0.95 (see supplemental Table 3 for all values). The average Cronbach's alpha for all cycles on the 22-item truncated MPAM-R was 0.90 (range 0.82-0.94) for enjoyment motives, 0.88 (range 0.82-0.92) for fitness motives, 0.90 (range 0.84-0.93) for competence motives, 0.92 (range 0.890.94 ) for appearance motives, and 0.85 (range $0.78-0.89$ ) for social motives. The average composite reliability for enjoyment motives was 0.88 (range 0.82-0.92), for fitness motives was 0.90 (range 0.830.94), for competence motives was 0.90 (range 0.85-0.93), for appearance motives was 0.92 (range 0.890.94 ), and for social motives was 0.86 (range $0.78-0.89$ ).

Longitudinal Invariance. Measurement invariance over time was tested with the truncated MPAM-R; though, due to computational limitations (e.g. convergence issues related to model complexity with 15 survey cycles and 75 latent variables) only mid-year measurements over the 5 years were used (one cycle per year). Results of the longitudinal invariance testing of the truncated MPAM-R suggest that the factor structure met the conditions for strong invariance (i.e., factor loadings and item intercepts constrained) (Table 2). [53] Strict invariance testing (i.e., factor loadings, item intercepts, and errors restrained) led to a change $>0.010$ in CFI and 0.015 in RMSEA and was not tenable. As strong invariance is considered the minimum to proceed with testing change over time, [54] analyses continued to the mixed effects regression models.

Trajectories of PA Motives. Mixed effects regression models to estimate PA motives trajectories revealed a statistically significant sex interaction for appearance and competence motives. Hence, the trajectories for all PA motives were sex stratified. For both sexes, mixed effects regression models revealed that four of the PA motives (i.e., fitness, competence, social, and appearance) followed a U-shape trajectory (Figure 2.a., and Figure 2.b.), whereas enjoyment motives followed a linear decrease for boys and girls (Table 3 ). Specifically, for boys, fitness motives decreased from cycle 1 to 12 and increased thereafter; competence motives decreased from cycle 1 to 9 and increased thereafter; social motives decreased from cycle 1 to 11 and increased thereafter; and appearance motives decreased from cycle 1 to 7 and increased thereafter. For girls, fitness motives decreased from cycle 1 to 11 and increased thereafter; competence motives decreased from cycle 1 to 9 and increased thereafter; social motives decreased from cycle 1 to 11 and increased thereafter; and appearance motives decreased from cycle 1 to 6 and increased thereafter.

Trajectories of MVPA. Mixed effects regression models to estimate MVPA trajectories revealed a statistically significant sex interaction. Hence, all mixed effects regression models were stratified by sex. For both girls and boys, MVPA followed an inverse U-shape peaking at cycle 7 for girls (mean age in years 
12.1; SD 0.6; range 11-14) and at cycle 9 for boys (mean age in years 12.8; SD 0.7; range 12-15) (Figure $3)$.

Trajectories of motives and MVPA. For boys, higher fitness motives at cycle 1 (intercept) were associated with lower initial MVPA levels. Increases in fitness motives were associated with lower MVPA levels (linear slope) and this negative relationship became more pronounced with higher levels of fitness motives (quadratic slope) (Table 4). In contrast, the model suggested that higher initial values of enjoyment and competence motives were related to higher levels of MVPA (intercepts) and that increases in these motives were positively associated with MVPA (linear slopes). For girls, the model suggested that initial level of enjoyment motives was positively related to childhood MVPA (intercept). The model also suggested that higher competence motives at cycle 1 were related to higher levels of MVPA (intercept), that increases in competence motives were predictive of increases in MVPA (linear slope) and that these increases were more pronounced at higher levels of competence motives (quadratic slope; Table 4).

\section{Discussion}

In this study, the longitudinal relationship of PA motives with self-report MVPA across 15 time points over a 5-year period spanning late childhood (10-11 years), early adolescence (12-14 years), and middle adolescence (15-16 years) was explored. Based on these findings, there are differences in how PA motives relate to MVPA by sex and developmental period. Specifically, fitness motives were negatively associated with boys' MVPA levels from late childhood to middle adolescence. In addition, enjoyment motives were positively associated with MVPA during late childhood for both sexes, and this positive association was maintained for boys until middle adolescence. Also, competence motives were positively associated with MVPA for both sexes from late childhood to middle adolescence, though it had a stronger positive relationship for girls in their middle adolescence. Furthermore, social and appearance motives for both sexes were not significantly related to MVPA during any developmental period examined.

Fitness Motives. Relationships between fitness motives and PA have been inconsistent in past research. $[32,35,36,38]$ Although our results are consistent with most previous studies showing that fitness motives are not related to girls participation in PA, $[32,35,38]$ our study adds to the literature by showing that for boys, greater fitness motives predict lower initial MVPA and are associated with decreasing MVPA over time. According to the authors of the MPAM-R, fitness motives are considered extrinsic forms of motivation and are not related to the maintenance of PA. [23] The current study is the first longitudinal study from late childhood to middle adolescence to provide empirical evidence that extrinsic forms of motivation are not just unrelated to PA, but they can be negatively associated with lower PA among boys. This suggests that fostering fitness motives would be ineffective at increasing MVPA in adolescents, despite having been identified and used as a motivational tool in previous PA promotion efforts. [35] The inability of young adolescents to truly consider the long-term consequences of a behavior may be a factor limiting the impact of fitness as a motive for PA. [35] By demonstrating a negative association with MVPA as early as in 9-10 years old boys, our findings lend further support that fitness concerns may only serve as PA in more mature individuals that have developed the cognitive processes required to realize 
the benefits of engaging in PA for fitness reasons. [55] Therefore, future studies may benefit from investigating how youth are interpreting fitness items, including whether boys and girls interpret these items similarly, to ensure accuracy in how researchers describe their results.

Enjoyment Motives. Others have argued that enjoyment motives should be the initial focus of any physical activity intervention. [56,57] Our results also highlight the need to ensure that PA interventions are enjoyable as greater enjoyment motives were strong predictors of more PA. These results are corroborated by several other studies. [31, 32, 34-36] However, previous studies, were unable to discern different patterns by sex in the relationship of enjoyment motives with the maintenance of PA behavior. Our results suggest that enjoyment motives are positively related to MVPA from age 9-16 years among boys; whereas among girls the positive relationship between enjoyment motives and MVPA was only noted during late childhood (9-10 years). A possible explanation for this result is that when girls are younger they display greater physical self-perception and as such they report greater enjoyment of PA which then reinforces their desire to participate in further PA. [58] However, as girls transition through puberty they may experience a degradation in their physical self-perception and a reduction in their enjoyment of PA. [58] Differences between our results and results from other publications [31, 32, 34-36] may be due to differences in research design (i.e., cross-sectional studies, short longitudinal studies) and different age groups (e.g., ages 7-12 years not encompassing the pubertal transition).

Competence Motives. Our results are in line with other research suggesting a positive association between competence motives and PA. [31, 32, 38] Our study demonstrated that greater competence motives for boys and girls, positively predicted self-reported MVPA from late childhood to middle adolescence. Additionally, higher levels of competence motives were predictive of more pronounced increases in girls' self-report MVPA over time. A systematic review that examined which factors contributed to drop out from organized sport among children and youth found that low perceptions of competence was one of the key factors influencing drop out. [59] Data from qualitative studies suggests that youth may feel embarrassed to participate in sports in front of their peers if they are not able to show that they are competent in the sport. [36] It is then possible that youth who engage in greater PA are driven by competence motives, and hence, maintain their PA participation. On the other hand, youth who do not hold high competence motives for PA participation are more susceptible to eventual barriers and are at a greater likelihood to discontinue their PA participation. Our study suggests that fostering competence motives in PA interventions may assist youth in adopting a PA mindset that will support their behavior for a longer term especially among girls, whom have been observed to drop out of PA much sooner and at faster rate than boys. [60]

Social Motives. Our results suggests that social motives were not associated with MVPA for both sexes. Previous investigations on this relationships have not been consistent with some showing that greater social motives were related to more PA, $[35,36]$ others indicated it was associated to less PA, [31] and some, like our study suggested it was not related to PA. [32,38] Our results are similar to the only other longitudinal study that examined the relationship of the five motives with objectively measured PA. [32] A recent systematic review of qualitative studies on barriers and facilitators of PA behavior in adolescents 
suggests that friendships have an influence on PA behavior. [61] However, researchers have found that youth tend to be friends with peers that accumulate similar levels of PA. [62] This might suggest that social motives are not helpful in the initiation of PA behavior, as youth choose friends that share certain behaviors, and not because these friends are going to make them start something new. Social motives may therefore not be key elements in the initiation and maintenance of PA.

Appearance Motives. Results from our work show that greater appearance motives were not related to MVPA, for either boys or girls. However, there were sex differences in the trajectories of appearance motives. In this study, both girls and boys identified appearance motives as their least important motives for taking part in PA throughout the study. Still, boys rated appearance motives higher than girls, but after turning 12 years old, the increase in the rating of this motive was more pronounced for girls than for boys. Previous cross-sectional publications have been inconsistent in their findings in relation to how appearance motives may relate to MVPA $[31,36]$ and a longitudinal study with two measurement points found no association between appearance motives and MVPA. [32] Collectively, this suggests that perceived and experienced social pressures to achieve a specific physique by both girls and boys do not lead to increased PA. [63-65] It is therefore recommended that interventions do not foster appearance motives as a tool to increase PA.

\section{Strengths and Limitations}

The comprehensive long-term and frequent record keeping in the MATCH study is a strength that should be acknowledged. This extensive data collection enabled the use of truncated MPAM-R items that were confirmed to be invariant across time to investigate changes in PA motives and PA from when children were 9-11 years until they were 15-16 years. Some limitations include the self-reporting of MVPA, which may over/under estimate PA. [66] These potential errors are nevertheless mitigated by the interest in describing general PA trends made possible with repeated measures, rather than an interest in reporting specific PA levels. [67] Moreover, computational challenges inhibited the estimation of longitudinal measurement invariance test using all available data. As such, despite showing invariance by using midyear measurements of every academic year, there may be some masked errors that could not be identified. Finally, the use of a truncated version of the MPAM-R may make other studies less comparable.

\section{Conclusions/public Health Implications}

With the objective to increase PA in adolescents, fostering motives centered on enjoyment and competence seem to be important intervention targets. Public health messages and interventions should place more importance on these motives to encourage older children and adolescents to adopt physically active lifestyles. Further, actions aimed at promoting MVPA should avoid promoting fitness motives to get older children and adolescents more active.

\section{List Of Abbreviations}


$\mathrm{PA}=$ Physical activity

MVPA = Moderate to vigorous physical activity

MPAM-R = Motives for physical activity measure revised

USA $=$ United States of America

$\mathrm{MATCH}=$ Monitoring Activities of Teenagers to Comprehend their Habits

CFA $=$ Confirmatory factor analysis

FIML = Full information maximum likelihood

RMSEA = Root mean square error of approximation

$\mathrm{CFI}=$ Comparative fit index

TLI = Tucker-Lewis index

\section{Declarations}

\section{Ethics approval and consent to participate}

All participants and their parents provided informed consent and the MATCH study was approved by the Comité d'Éthique de la Recherche du Centre Hospitalier de l'Université de Sherbrooke (11-025-M9).

\section{Consent for publication}

Not applicable

\section{Availability of data and materials}

The original data analyzed for these analyses are available through a data sharing agreement with the MATCH study research team. More information on this may be obtained from the principal investigator of the MATCH study, Dr. Mathieu Belanger.

\section{Competing interests}

The authors declare that they have no competing interests

\section{Funding}

The MATCH project is supported by the New Brunswick Health Research Foundation (\#20130729), by the Social Sciences and Humanities Research Council (\#435-2016-0888) and by Sport Canada through the joint Sport Participation Research Initiative (\#862-2010-0001; \#862-2014-0002). 


\section{Authors' contributions}

PAN conceptualized the objectives of the analysis, designed and conducted statistical analyses, and wrote the manuscript. JG helped with writing the introduction, discussion, and critically reviewed all other content. JB, KG and ID helped with the development of the analysis plan, data interpretation and critically reviewed and edited all other content. CS helped with interpretation of data and critically reviewed all other content. JB helped with writing the introduction and critically reviewed all other content. MB discussed objectives, data analysis plan, contributed to interpreting results and critically reviewed the manuscript. MB is the principal investigator of the MATCH study.

\section{Acknowledgements}

During the development of this manuscript PAN held a postdoctoral fellowship from New Brunswick Health Research Foundation \& Canadian Institute of Health Reseach- Strategy for Patiented Oriented Research- Maritime SPOR Support Unit.

\section{References}

1. Bailey D, Boddy L, Savory L, Denton S, Kerr C. Associations between cardiorespiratory fitness, physical activity and clustered cardiometabolic risk in children and adolescents: the HAPPY study. Eur J Pediatr. 2012;171:1317-23.

2. Bloemers F, Collard D, Paw M, Van Mechelen W, Twisk J, Verhagen E. Physical inactivity is a risk factor for physical activity-related injuries in children. Br J Sports Med. 2011;46:669-74.

3. Janssen I, Leblanc A. Systematic review of the health benefits of physical activity and fitness in school-aged children and youth. Int J Behav Nutr Phys Act. 2010;7:40.

4. Tremblay M, LeBlanc A, Kho M, Saunders T, Larouche R, Colley R, et al. Systematic review of sedentary behaviour and health indicators in school-aged children and youth. Int $\mathrm{J}$ Behav Nutr Phys Act. 2011;8:98.

5. Poitras VJ, Gray CE, Borghese MM, Carson V, Chaput J-P, Janssen I, et al. Systematic review of the relationships between objectively measured physical activity and health indicators in school-aged children and youth ${ }^{1}$. Appl Physiol Nutr Metab. 2016;41 6 (Suppl. 3):S197-239. doi:10.1139/apnm2015-0663.

6. World Health Organization (WHO). Physical activity. 2017.

http://www.who.int/topics/physical_activity/en/. Accessed 29 Jan 2018.

7. World Health Organization (WHO). 10 Facts on Physical Activity. 2014,:2016. http://www.who.int/features/factfiles/physical_activity/en/. Accessed 29 Jan 2018.

8. Mintjens S, Menting MD, Daams JG, van Poppel MNM, Roseboom TJ, Gemke RJBJ. Cardiorespiratory Fitness in Childhood and Adolescence Affects Future Cardiovascular Risk Factors: A Systematic Review of Longitudinal Studies. Sport Med. 2018;48:2577-605. doi:10.1007/s40279-018-0974-5. 
9. Ruiz JR, Castro-Piñero J, Artero EG, Ortega FB, Sjöström M, Suni J, et al. Predictive validity of healthrelated fitness in youth: A systematic review. Br J Sports Med. 2009;43:909-23.

10. Colley RC, Carson V, Garriguet D, Janssen I, Roberts KC, Tremblay MS. Physical activity of Canadian children and youth. Heal Reports. 2017;28:8-16. doi:October 2017.

11. van Sluijs E, McMinn A, Griffin S. Effectiveness of interventions to promote physical activity in children and adolescents: systematic review of controlled trials. BMJ. 2007;335:703.

12. Metcalf B, Henley W, Wilkin T. Republished research: Effectiveness of intervention on physical activity of children: systematic review and meta-analysis of controlled trials with objectively measured outcomes. Br J Sports Med. 2013;47:226.

13. Dobbins M, Husson H, Decorby K, RI L. School-based physical activity programs for promoting physical activity and fitness in children and adolescents aged 6 to 18. 2013;18.

14. Dudley D, Okely A, Pearson P, Cotton W. A systematic review of the effectiveness of physical education and school sport interventions targeting physical activity, movement skills and enjoyment of physical activity. Eur Phys Educ Rev. 2011;17:353-78.

15. Nader PR, Bradley RH, Houts RM, McRitchie SL, O’Brien M. Moderate-to-vigorous physical activity from ages 9 to 15 years. Jama. 2008;300:295-305. http://archderm.jamanetwork.com/data/Journals/JAMA/4424/joc80065_295_305.pdf.

16. Corder K, Winpenny E, Love R, Brown HE, White M, Sluijs E Van. Change in physical activity from adolescence to early adulthood: A systematic review and meta-analysis of longitudinal cohort studies. British Journal of Sports Medicine. 2019;53:496-503. doi:10.1136/bjsports-2016-097330.

17. Ajzen I. The theory of planned behavior. Organ Behav Hum Decis Process. 1991;50:179-211.

18. Bronfenbrenner U. Ecological models of human development. In: Readings on the development of children. 2nd ed. Oxford: Elsevier; 1994. p. 37-43.

19. Bandura A. Self-Efficacy. The Corsini Encyclopedia of Psychology. 2010;:1-3. doi:doi:10.1002/9780470479216.corpsy0836.

20. Ryan RM, Deci EL. Self-determination theory and the facilitation of intrinsic motivation, social development, and well-being. Am Psychol. 2000;55:68-78.

21. Wigfield A, Eccles JS. Expectancy-value theory of achievement motivation. Contemp Educ Psychol. 2000;25:68-81.

22. Sabiston CM, Pila E, Vani M, Thogersen-Ntoumani C. Body image, physical activity, and sport: $A$ scoping review. Psychol Sport Exerc. 2019;42:48-57.

23. Ryan RM, Fredrick CM, Lepes D, Rubio N, Sheldon KM. Intrinsic Motivation and Exercise Adherence. International Journal of Sport Psychology. 1997;28:335-54.

24. McLachlan S, Hagger M. Do people differentiate between intrinsic and extrinsic goals for physical activity? J Sport Exerc Psychol. 2011;33:273-88.

25. Sebire SJ, Standage M, Vansteenkiste M. Examining intrinsic versus extrinsic exercise goals: Cognitive, affective, and behavioral outcomes. J Sport Exerc Psychol. 2009;31:189-210. 
26. Teixeira PJ, Carraça E V., Markland D, Silva MN, Ryan RM. Exercise, physical activity, and selfdetermination theory: A systematic review. 2012. doi:10.1186/1479-5868-9-78.

27. Frederick CM, Ryan RM. Differences In Motivation for Sport and Exercise and their Relations with Participation and Mental Health. Journal of Sport Behavior. 1993;16:125-45.

28. B. Owen K, Smith J, Lubans DR, Ng JYY, Lonsdale C. Self-determined motivation and physical activity in children and adolescents: A systematic review and meta-analysis. Prev Med (Baltim). 2014;67:270-9. doi:10.1016/j.ypmed.2014.07.033.

29. Gillison FB, Standage M, Skevington SM. Relationships among adolescents' weight perceptions, exercise goals, exercise motivation, quality of life and leisure-time exercise behaviour: A selfdetermination theory approach. Health Educ Res. 2006;21:836-47. doi:10.1093/her/cyl139.

30. Chicote-López J, Abarca-Sos A, Gallardo LO, García-González L. Social antecedents in physical activity: Tracking the self-determination theory sequence in adolescents. $\mathrm{J}$ Community Psychol. 2018;46:356-73.

31. Seghers J, Vissers N, Rutten C, Decroos S, Boen F. Intrinsic goals for leisure-time physical activity predict children's daily step counts through autonomous motivation. Psychol Sport Exerc. 2014;15:247-54. doi:10.1016/j.psychsport.2014.01.003.

32. Dishman RK, Saunders RP, Mclver KL, Dowda M, Pate RR. Construct Validity of Selected Measures of Physical Activity Beliefs and Motives in Fifth and Sixth Grade Boys and Girls. J Pediatr Psychol. 2013;38:563-76. doi:10.1093/jpepsy/jst013.

33. Goguen Carpenter J, Bélanger M, O'Loughlin J, Xhignesse M, Ward S, Caissie I, et al. Association between physical activity motives and type of physical activity in children. Int J Sport Exerc Psychol. 2015;:1-15. doi:10.1080/1612197X.2015.1095779.

34. Woods AM, Bolton KN, Graber KC, Crull GS. Influences of perceived motor competence and motives on children's physical activity. J Teach Phys Educ. 2007;26:390-403. doi:Article.

35. Iannotti RJ, Chen R, Kololo H, Petronyte G, Haug E, Roberts C. Motivations for Adolescent Participation in Leisure-Time Physical Activity: International Differences. J Phys Act Heal. 2012;9:106-14. www.JPAH-Journal.com. Accessed 23 Jul 2018.

36. Gillison F, Osborn M, Standage M, Skevington S. Exploring the experience of introjected regulation for exercise across gender in adolescence. Psychol Sport Exerc. 2009;10:309-19. doi:10.1016/j.psychsport.2008.10.004.

37. Allender S, Cowburn G, Foster C. Understanding participation in sport and physical activity among children and adults: a review of qualitative studies. Health Educ Res. 2006;21:826-35. doi:10.1093/her/cyl063.

38. Kopcakova J, Veselska ZD, Geckova AM, Kalman M, van Dijk JP, Reijneveld SA. Do motives to undertake physical activity relate to physical activity in adolescent boys and girls? Int J Environ Res Public Health. 2015;12:7656-66.

39. Bagøien TE, Halvari $H$, Nesheim H. Self-determined motivation in physical education and its links to motivation for leisure-time physical activity, physical activity, and well-being in general. Percept Mot 
Skills. 2010;111:407-32.

40. Markland D, Tobin V. A modification to the behavioural regulation in exercise questionnaire to include an assessment of amotivation. J Sport Exerc Psychol. 2004;26:191-6. doi:10.1123/jsep.26.2.191.

41. Fenton SAM, Duda JL, Barrett T. Optimising physical activity engagement during youth sport: a selfdetermination theory approach. J Sports Sci. 2016;34:1874-84.

doi:10.1080/02640414.2016.1142104.

42. Bélanger M, Caissie I, Beauchamp J, O'Loughlin J, Sabiston C, Mancuso M. Monitoring activities of teenagers to comprehend their habits: study protocol for a mixed-methods cohort study. BMC Public Health. 2013;13:649. doi:10.1186/1471-2458-13-649.

43. Bélanger M, Gray-Donald K, O'loughlin J, Paradis G, Hanley J. Influence of Weather Conditions and Season on Physical Activity in Adolescents. Ann Epidemiol. 2009;19:180-6. doi:10.1016/j.annepidem.2008.12.008.

44. Prochaska JJ, Sallis JF, Long B. A physical activity screening measure for use with adolescents in primary care. Arch Pediatr Adolesc Med. 2001;155:554-9. doi:10.1001/archpedi.155.5.554.

45. Biddle SJ, Gorely T, Pearson N, Bull FC. An assessment of self-reported physical activity instruments in young people for population surveillance: Project ALPHA. Int J Behav Nutr Phys Act. 2011;8:1. doi:10.1186/1479-5868-8-1.

46. Hardie Murphy M, Rowe DA, Belton S, Woods CB. Validity of a two-item physical activity questionnaire for assessing attainment of physical activity guidelines in youth. BMC Public Health. 2015;15. doi:10.1186/s12889-015-2418-6.

47. Sabiston CM, Crocker PRE. Exploring Self-Perceptions and Social Influences as Correlates of Adolescent Leisure-Time Physical Activity. J Sport Exerc Psychol. 2008;30:3-22. http://journals.humankinetics.com/doi/pdf/10.1123/jsep.30.1.3. Accessed 20 Jun 2017.

48. Norman G, Streiner D. Biostatistics: the bare essentials. 3rd Ed. Hamilton, ON: B.C. Decker; 2008.

49. StataCorp. Stata Statistical Software. 2017.

50. Raykov T. Estimation of composite reliability for congeneric measures. Appl Psychol Meas. 1997;21:173-84.

51. Widaman KF, Ferrer E, Conger RD. Models: Measuring the Same Construct across Time. Child Dev Perspect. 2011;4:10-8.

52. Muthén LK, Muthén BO. Mplus User's Guide. 7th edition. Los Angeles,CA: Muthén \& Muthén. www.StatModel.com. Accessed 26 Oct 2019.

53. Chen FF. Sensitivity of goodness of fit indexes to lack of measurement Invariance sensitivity of goodness of fit indexes to lack of measurement invariance. Struct Equ Model. 2007;14:464-504. doi:10.1080/10705510701301834.

54. Cheung GW, Rensvold RB. Evaluating Goodness-of- Fit Indexes for Testing Measurement Invariance. Struct Equ Model A Multidiscip J. 2002;9:233-55. doi:10.1207/S15328007SEM0902. 
55. Johnson SB, Blum RW, Giedd JN. Adolescent Maturity and the Brain: The Promise and Pitfalls of Neuroscience Research in Adolescent Health Policy. J Adolesc Heal. 2009;45:216-21. doi:10.1016/j.jadohealth.2009.05.016.

56. van Sluijs EMF, Kriemler S. Reflections on physical activity intervention research in young people dos, don'ts, and critical thoughts. Int J Behav Nutr Phys Act. 2016;13:25. doi:10.1186/s12966-0160348-z.

57. Lewis BA, Williams DM, Frayeh A, Marcus BH. Self-efficacy versus perceived enjoyment as predictors of physical activity behaviour. Psychol Heal. 2016;31:456-69.

doi:10.1080/08870446.2015.1111372.

58. Labbrozzi D, Robazza C, Bertollo M, Bucci I, Bortoli L. Pubertal development, physical self-perception, and motivation toward physical activity in girls. J Adolesc. 2013;36:759-65.

doi:10.1016/j.adolescence.2013.06.002.

59. Crane J, Temple V. A systematic review of dropout from organized sport among children and youth. Eur Phys Educ Rev. 2015;21:114-31. doi:10.1177/1356336X14555294.

60. Chung AE, Skinner AC, Steiner MJ, Perrin EM. Physical Activity and BMI in a Nationally Representative Sample of Children and Adolescents. Clin Pediatr (Phila). 2012;51:122-9. doi:10.1177/0009922811417291.

61. Martins J, Marques A, Sarmento H, Carreiro Da Costa F. Adolescents' perspectives on the barriers and facilitators of physical activity: A systematic review of qualitative studies. Health Educ Res. 2015;30:742-55. doi:10.1093/her/cyv042.

62. de la Haye K, Robins G, Mohr P, Wilson C. How physical activity shapes, and is shaped by, adolescent friendships. Soc Sci Med. 2011;73:719-28. doi:10.1016/J.SOCSCIMED.2011.06.023.

63. GROGAN S, RICHARDS H. Focus Groups with Boys and Men. Men Masc. 2002;4:219-32. doi:10.1177/1097184X02004003001.

64. Niven A, Fawkner S, Knowles AM, Henretty J, Stephenson C. Social physique anxiety and physical activity in early adolescent girls: The influence of maturation and physical activity motives. J Sports Sci. 2009;27:299-305. doi:10.1080/02640410802578164.

65. Sampasa-Kanyinga H, Hamilton HA, Willmore J, Chaput JP. Perceptions and attitudes about body weight and adherence to the physical activity recommendation among adolescents: the moderating role of body mass index. Public Health. 2017;146:75-83. doi:10.1016/j.puhe.2017.01.002.

66. Loprinzi PD, Cardinal BJ, Article R. Measuring Children's Physical Activity and Sedentary Behaviors. J Exerc Sci Fit. 2011;9:15-23. doi:10.1016/S1728-869X(11)60002-6.

67. Levin S, Jacobs DR, Ainsworth BE, Richardson MT, Leon AS. Intra-individual variation and estimates of usual physical activity. Ann Epidemiol. 1999;9:481-8. doi:10.1016/S1047-2797(99)00022-8.

\section{Tables}

Due to technical limitations, tables are available in the Supplementary Files below. 

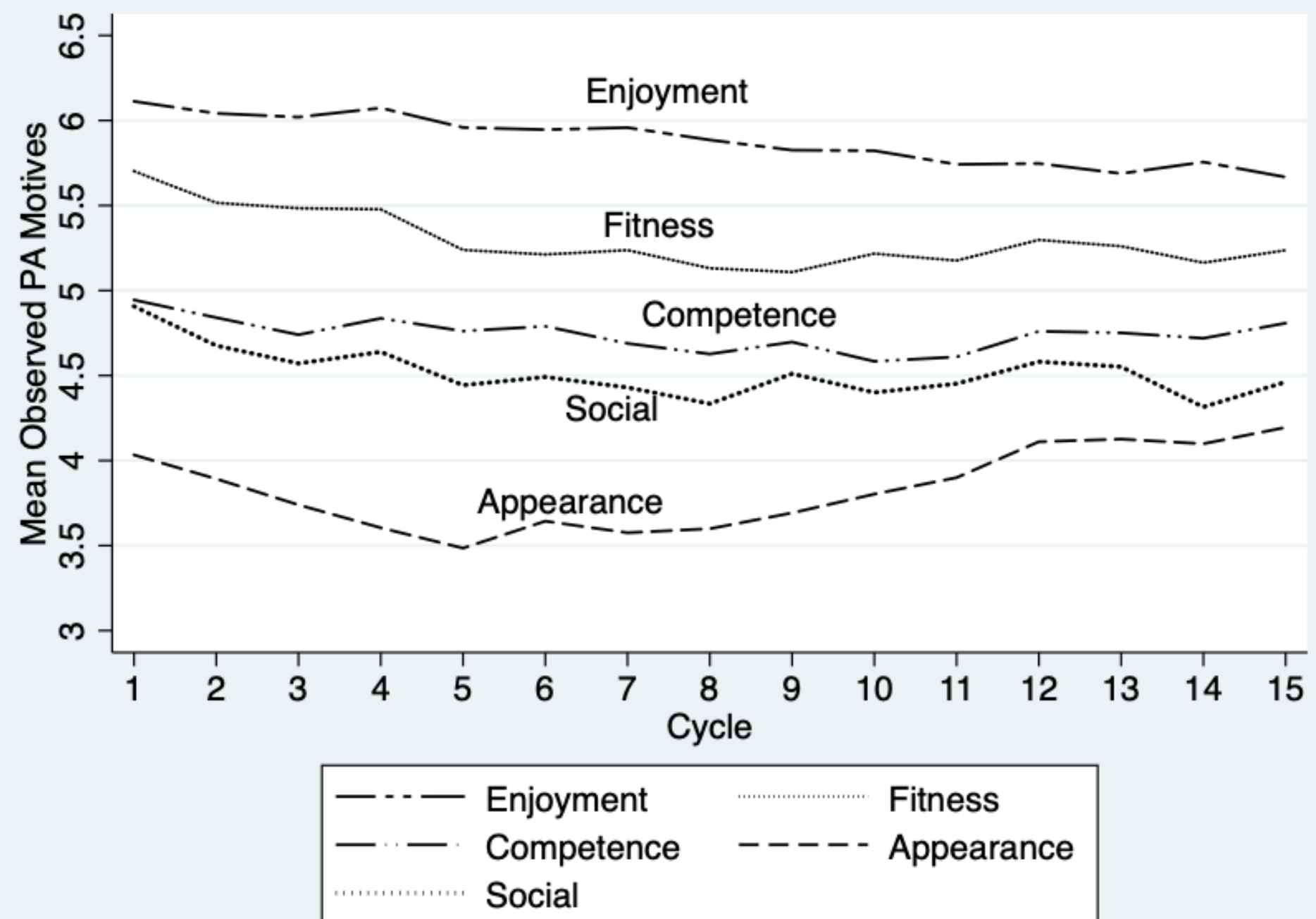

\section{Figure 1}

Mean of overall Observed Physical Activity Motives over Fifteen Survey Cycles
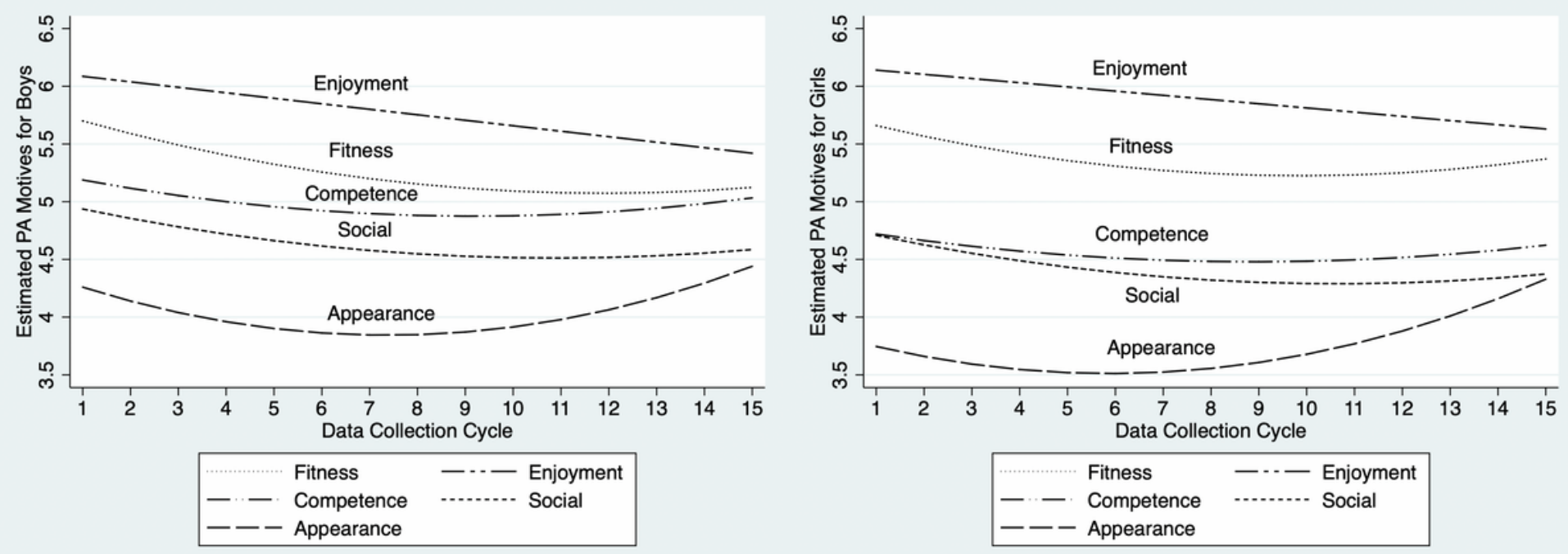
Figure 2

Sex-Stratified Estimated Physical Activity Motives over Fifteen Survey Cycles 2.a. Estimated PA Motives for Boys. 2.b. Estimated PA Motives for Girls

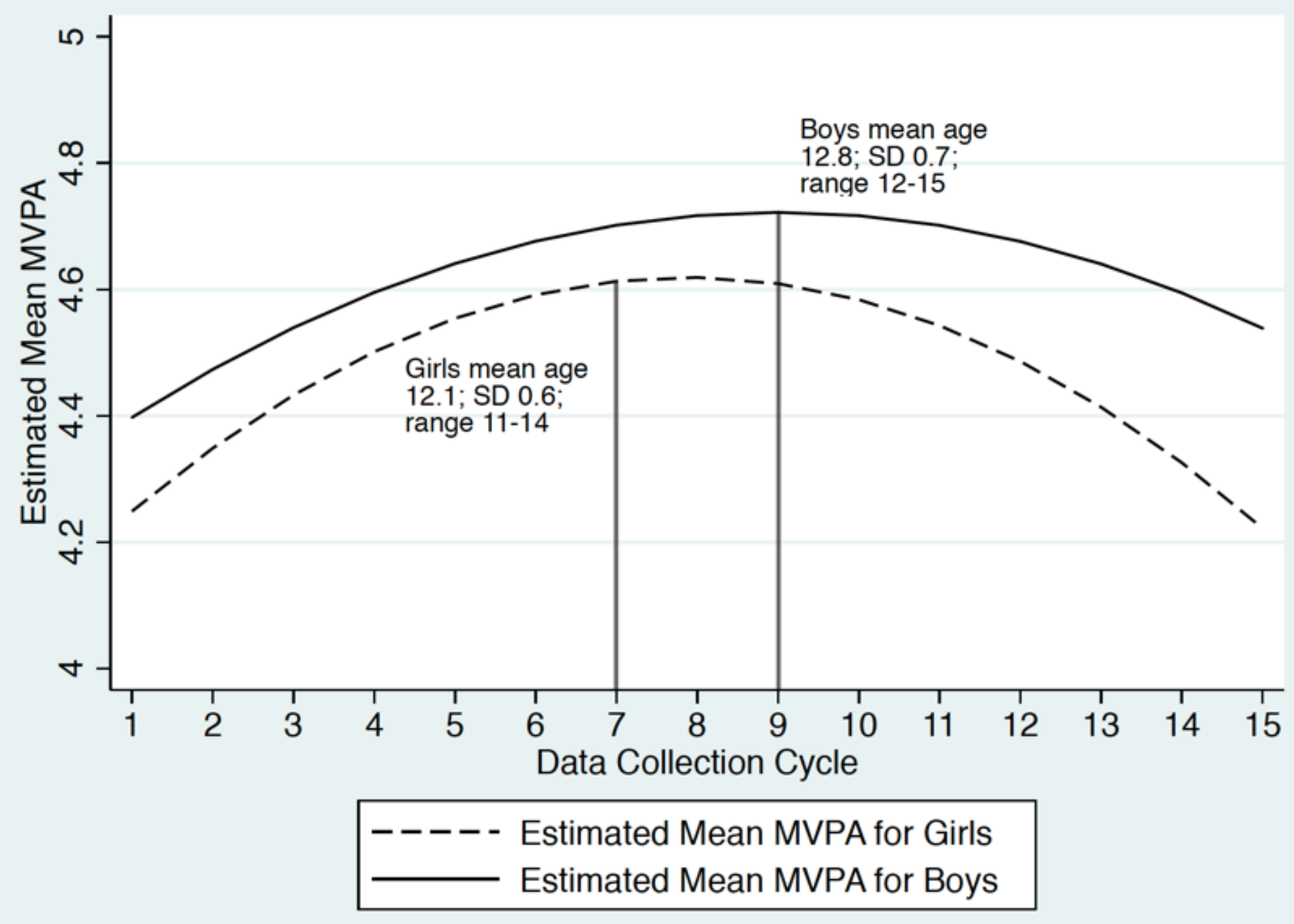

Figure 3

Estimated Mean MVPA* for Boys and Girls from the Sex-Stratified Mixed Effects Regression Model with MVPA Predicted by PA Motives over Fifteen Survey Cycles (5 years). *MVPA: is the average number of days per week in which a participant reports engaging in at least 60 minutes of moderate to vigorous physical activity

\section{Supplementary Files}

This is a list of supplementary files associated with this preprint. Click to download.

- AbiNaderMotivesSuppTables20191129.docx

- AbiNaderMotivesTables20191129.docx 